\title{
CRESCIMENTO DE ABOBRINHA ITALIANA (Cucurbita pepo L.) CULTIVADO COM SOLO ENRIQUECIDO COM LODO DE ESGOTO
}

\author{
Nathália Aparecida Bragança Fávaris ${ }^{1}$ \\ Allan Rocha de Freitas ${ }^{2}$ \\ Carla Basilio Monteiro ${ }^{3}$ \\ Manoel Victor Borges Pedrosa ${ }^{4}$ \\ José Carlos Lopes ${ }^{5}$
}

Resumo: No Brasil, a abobrinha italiana é uma hortaliça de grande aceitação e de elevada importância econômica. O objetivo do trabalho foi avaliar o crescimento da abobrinha italiana em solo enriquecido com lodo de esgoto. O estudo foi conduzido, em casa de vegetação do Laboratório de Análise de Sementes do Centro de Ciências Agrárias e Engenharias da Universidade Federal do Espírito Santo, em Alegre-ES. Foram utilizadas sementes de abobrinha italiana (Cucurbita pepo L.) em três substratos compostos por Latossolo Vermelho Amarelo distrófico, areia lavada, e lodo de esgoto nas concentrações de 0; $40 ; 80$ e 120 t ha $^{-1}$, em delineamento experimental de blocos casualizados. Foram analisados: número de folhas, comprimento de haste $(\mathrm{cm})$ e comprimento da primeira folha $(\mathrm{cm})$. Verificou-se que a concentração de 205, 6 t ha ${ }^{-1}$ de lodo de esgoto proporciona maior desenvolvimento das plantas. Os maiores valores médios de crescimento foram obtidas após 60 dias da emergência.

Palavras-chave: Cucubitaceae; Biossólido; Substrato.

\footnotetext{
${ }^{1}$ Departamento de Produção Vegetal do Centro de Ciências Agrárias e Engenharias da Universidade Federal do Espírito Santo (CCAE-UFES), Alto Universitário, Brasil. E-mail: nath-braganca@hotmail.com.

2 Departamento de Produção Vegetal do Centro de Ciências Agrárias e Engenharias da Universidade Federal do Espírito Santo (CCAE-UFES), Alto Universitário, Brasil. E-mail: allanrochaf@gmail.com.

3 Departamento de Produção Vegetal do Centro de Ciências Agrárias e Engenharias da Universidade Federal do Espírito Santo (CCAE-UFES), Alto Universitário, Brasil. E-mail: carlabasilio92@yahoo.com.br.

4 Departamento de Produção Vegetal do Centro de Ciências Agrárias e Engenharias da Universidade Federal do Espírito Santo (CCAE-UFES), Alto Universitário, Brasil. E-mail: victorpedrosa2009@hotmail.com.

5 Departamento de Produção Vegetal do Centro de Ciências Agrárias e Engenharias da Universidade Federal do Espírito Santo (CCAE-UFES), Alto Universitário, Brasil. E-mail: jcufes@bol.com.br.
} 